\title{
The Fine Structure of Smooth Muscle in Bipalium Kewense Moseley, Its Possible Relation to Evolution of Skeletal Muscle
}

\author{
C. N. Sun, S. B. Cheng Chew and H. J. White \\ Veterans Administration Hospital and Department of Pathology, \\ University of Arkansas for Medical Sciences, \\ Little Rock, Arkansas 72206. U.S.A.
}

Received June 21, 1977

The structure and function of muscle and its contraction mechanism have been extensively studied in the skeletal muscles of vertebrate animals. The cross-striated muscle fibers, which are composed of thick and thin myofilaments, are cross-linked. The smooth muscles of invertebrates also contain cross-linked thick and thin myofilaments but they are not arranged in crossed bands as in striated fibers (Hanson and Lowy 1960, MacRae 1963). The structure of the dense body consistently present in the invertebrates smooth muscle cells has been suggested as having properties of Z-band (Hanson and Lowy 1961, MacRae 1963).

The general morphology of the muscles of a terrestrial flatworm, Bipalium Kewense, is very much like the smooth muscle of some other invertebrates. However, they are composed of the thick, intermediate and thin myofilaments rather than thick and thin filaments, as seen in the molluscan muscle (North 1963, Philpott et al. 1960), as well as in the planarian pharyngeal muscle (MacRae 1965). The arrangement of myofilaments, dense bodies and sarcoplasmic reticulum is presented here.

\section{Materials and methods}

The organism in this study is a land Turbellarian, Bipalium Kewense. The animal was cut into small pieces about $1 \mathrm{~mm}^{3}$ and immediately fixed in $4 \%$ glutaraldehyde in phosphate buffer for two hours, then post-fixed in $1 \%$ buffered osmium tetroxide for one hour. After dehydration, tissues were embedded in Epon 812. Thin sections were stained with uranyl acetate and lead citrate.

\section{Results}

Muscle fibers and myofilaments:

There are three types of myofilaments within the muscle fiber or myofiber which are parallel to the long axis of the fiber (Figs. 1,2). These are the thick, intermediate and the thin myofilaments. It is very difficult to obtain a cross section of the thin filaments, because in most cases the thin filaments are obliquely cut. The thick filaments are distributed in a relatively regular-spaced fashion. In some 


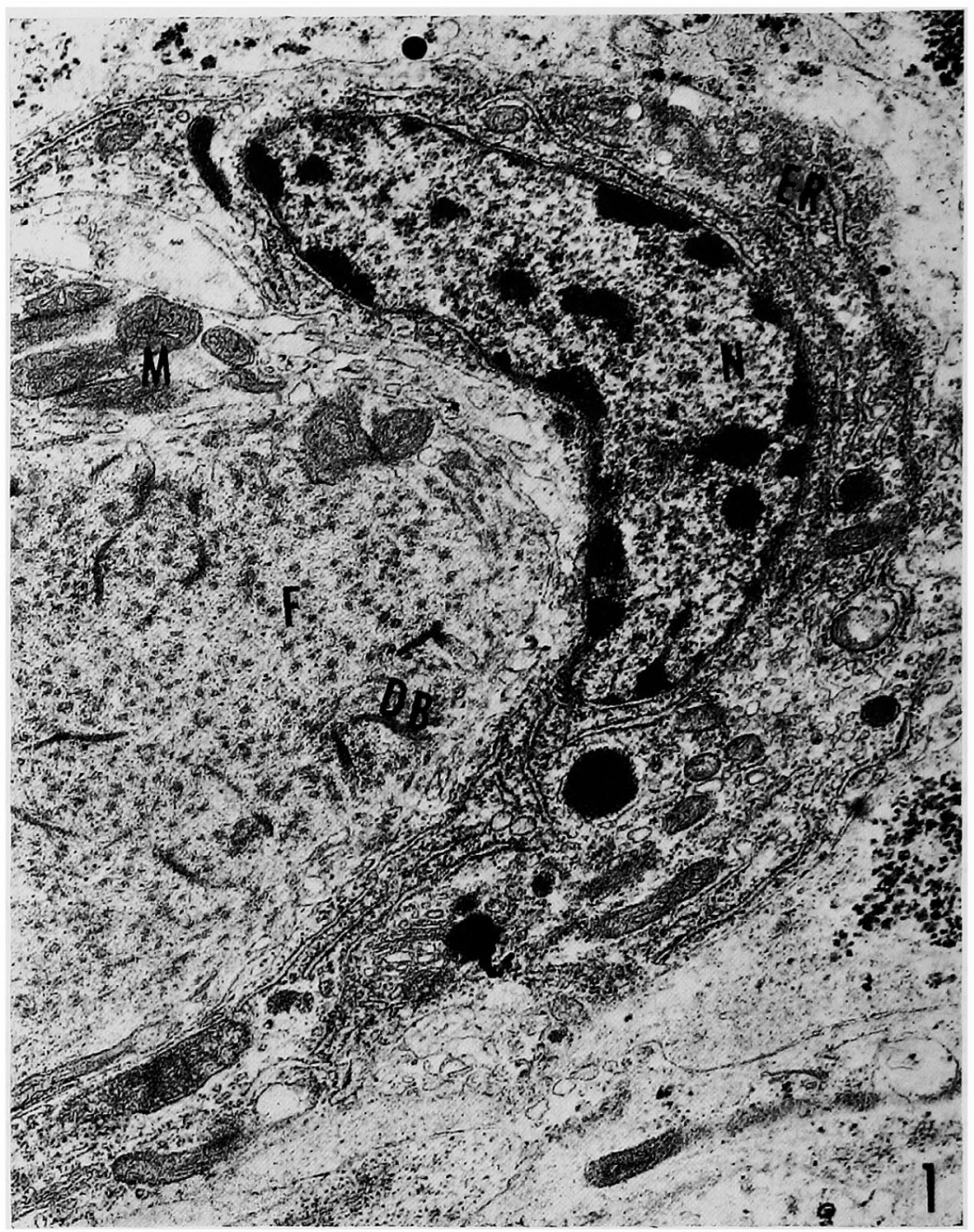

Fig. 1. Cross section of a smooth muscle cell to show the nucleus (N), mitochondria (M), endoplasmic reticulum (ER), myofilaments (F) and dense bodies (DB). $\quad \times 16,000$.

areas (Fig. 3, arrow), approximately 8-10 intermediate myofilaments in cross section surround a single thick myofilament. Whether this be an usual pattern cannot be confirmed. The diameter of the thick filaments range from $400-600 \AA$, the diameter of intermediate filaments range from $80-120 \AA$, and the diameter of thin filaments range from $50-60 \AA$. Further, the thick filaments are in turn composed of many fine fibrillary structures and range from $1.4-3 \mu \mathrm{m}$ in length. The intermediate and thin filaments are associated with dense bodies.

The sarcotubular system and the dense bodies:

The sarcotubular system or the sarcoplasmic reticulum system that appears in the muscles studied is quite unique. It is composed of a tubular system that runs across the periphery of the myofiber in longitudinal section. This tubular sarcoplasmic is reticulum very closely related to the sarcolemma as well as the dense bodies (Figs. 4, 5). Tubular cisternae run in-between the myofilaments (Fig. 2). 


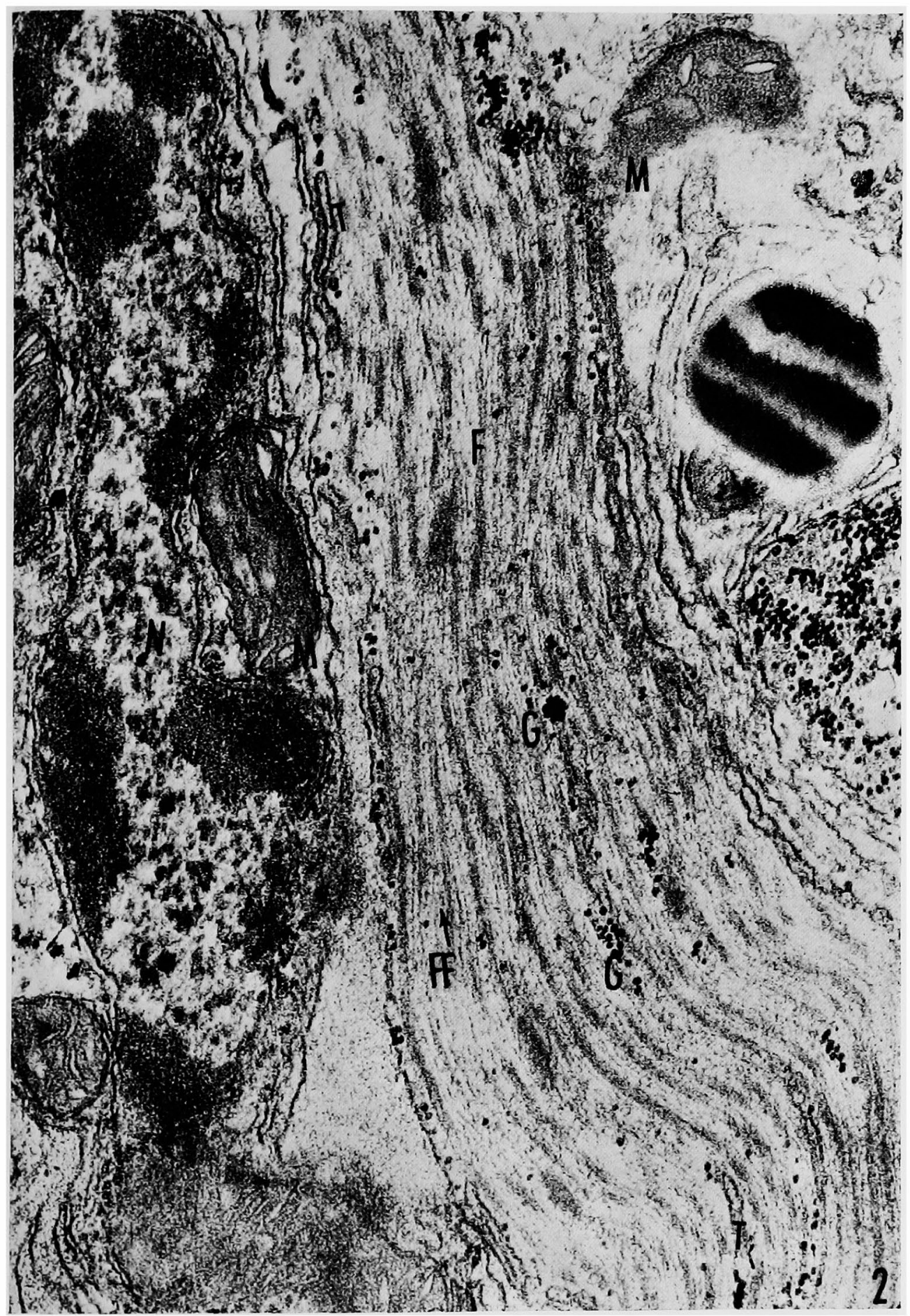

Fig. 2. Longitudinal section of a smooth muscle cell to show the nucleus (N), mitochondria (M), thick filaments $(\mathrm{F})$, intermediate filaments $(\mathrm{FF})$, glycogen granules in single or aggregates $(\mathrm{G})$ and tubular sarcoplasmic reticulum $(\mathrm{T}) . \times 50,000$. 

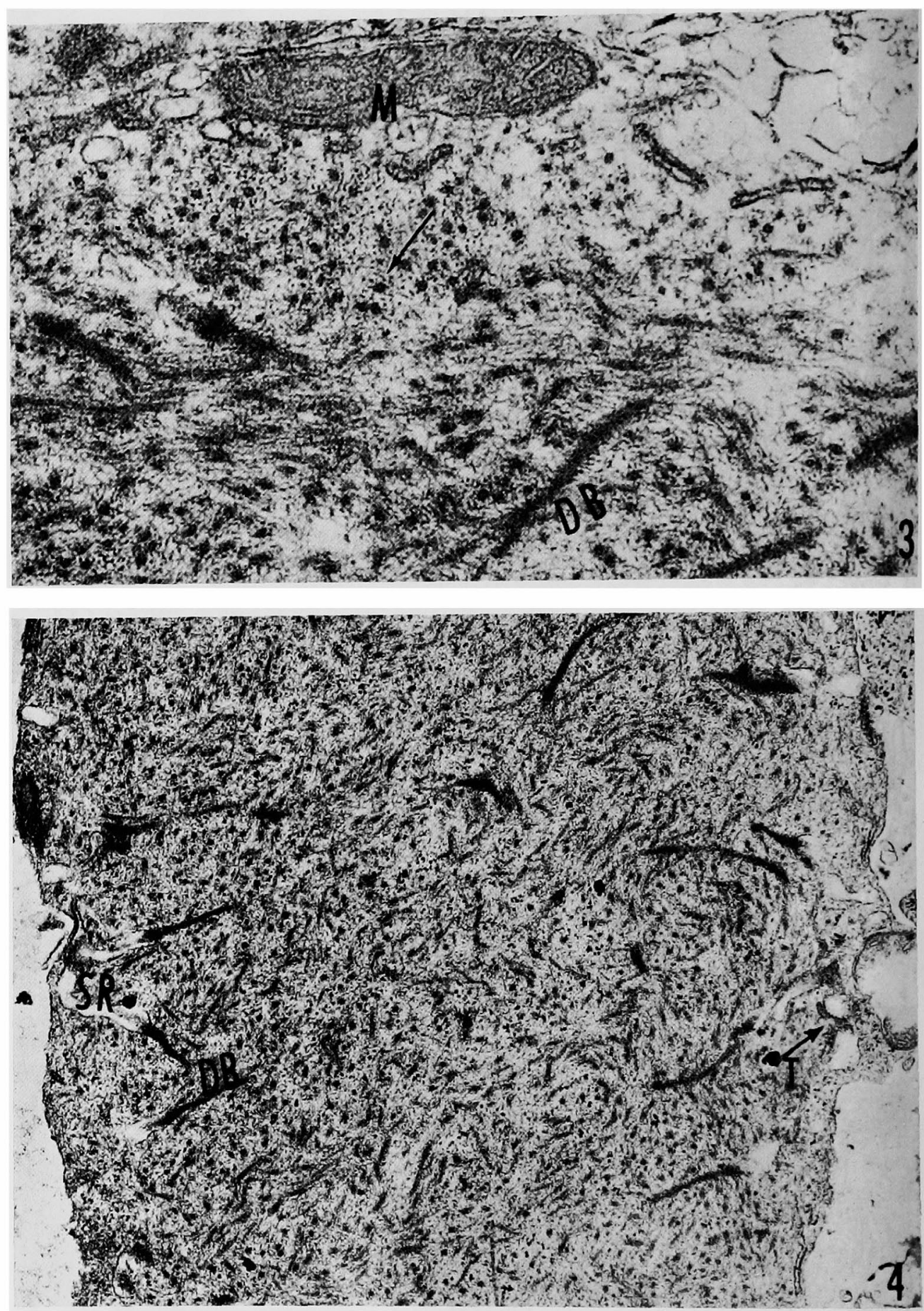

Figs. 3-4. 3, cross section of a portion of smooth muscle cell to show 8-10 intermediate filaments surrounding a single thick filament $(\rightarrow)$. DB, dense body; M, mitochondrion. $\times 38,000.4$, cross section of a portion of a smooth muscle cell to show myofilaments, dense bodies (DB), sarcoplasmic reticulum (SR) and tubular sarcoplasmic reticulum $(T) . \quad \times 20,000$. 

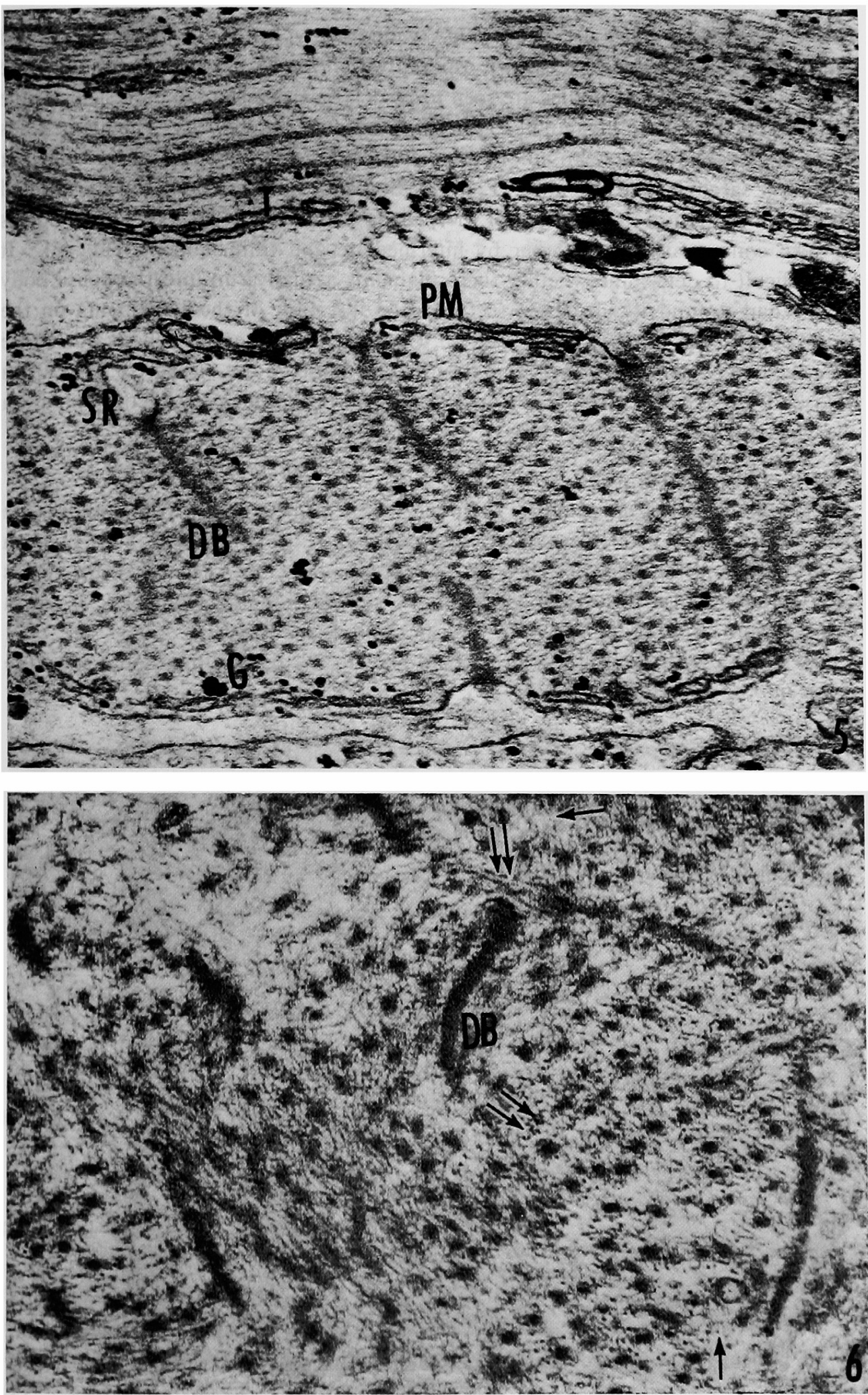

Figs. 5-6. 5, longitudinal and oblique sections of smooth muscle cells to show plasma membrane (PM), dense bodies (DB), glycogen granules $(G)$, sarcoplasmic reticulum (SR) and tubular sarcoplasmic reticulum (T). $\quad 50,000$. 6, cross and oblique sections of a portion of a smooth muscle cell to show thick, intermediate (double arrow) and thin (arrow) myofilaments. Intermediate and thin filaments are connected to dense bodies (DB) 
The longitudinal tubules are about $500 \AA$ wide and typically are closely associated with dense bodies, which are shown especially well in the transverse section of the muscle (Fig. 4). Dense bodies are seen to associate with cisternae or vesicles of the sarcoplasmic reticulum invaginated from the edge of the myofiber. The filamentous dense bodies tend to have an amorphous extension inside the space of the vesicular or tubular sarcoplasmic reticulum (Fig. 4). Some of the dense bodies are associated with an invagination or infolding of the plasma membrane (Fig. 5). Dense bodies are also connected to each other by intermediate and thin filaments (Fig. 6). It was difficult to determine whether these filaments actually run into the dense body rather than around it. In some sections, the insertion of these filaments on the dense bodies was more evident (Fig. 6). In this case, the amorphous substance from the body deposits at the invagination. The dense bodies measure $0.06-0.11 \mu \mathrm{m}$ wide and $0.5-1.1 \mu \mathrm{m}$ long. They are similar to those appearing in the planarian pharyngeal muscle (MacRae 1965).

Nucleus and mitochondria:

The nucleus is situated in the periphery of the muscle cell. Mitochondria are also located mostly in the periphery of the myofiber (Fig. 1). Occasionally one can see a mitochondrion situated between the myofilaments, but such an occurrence is rare. Mitochondria are round, oval, or rod-shaped, ranging from $0.6-2.0 \mu \mathrm{m}$ in diameter, and dense granules can be seen in their cristae (Fig. 7).

A schematic drawing is constructed to explain the three dimensional structure of the smooth muscle fiber.

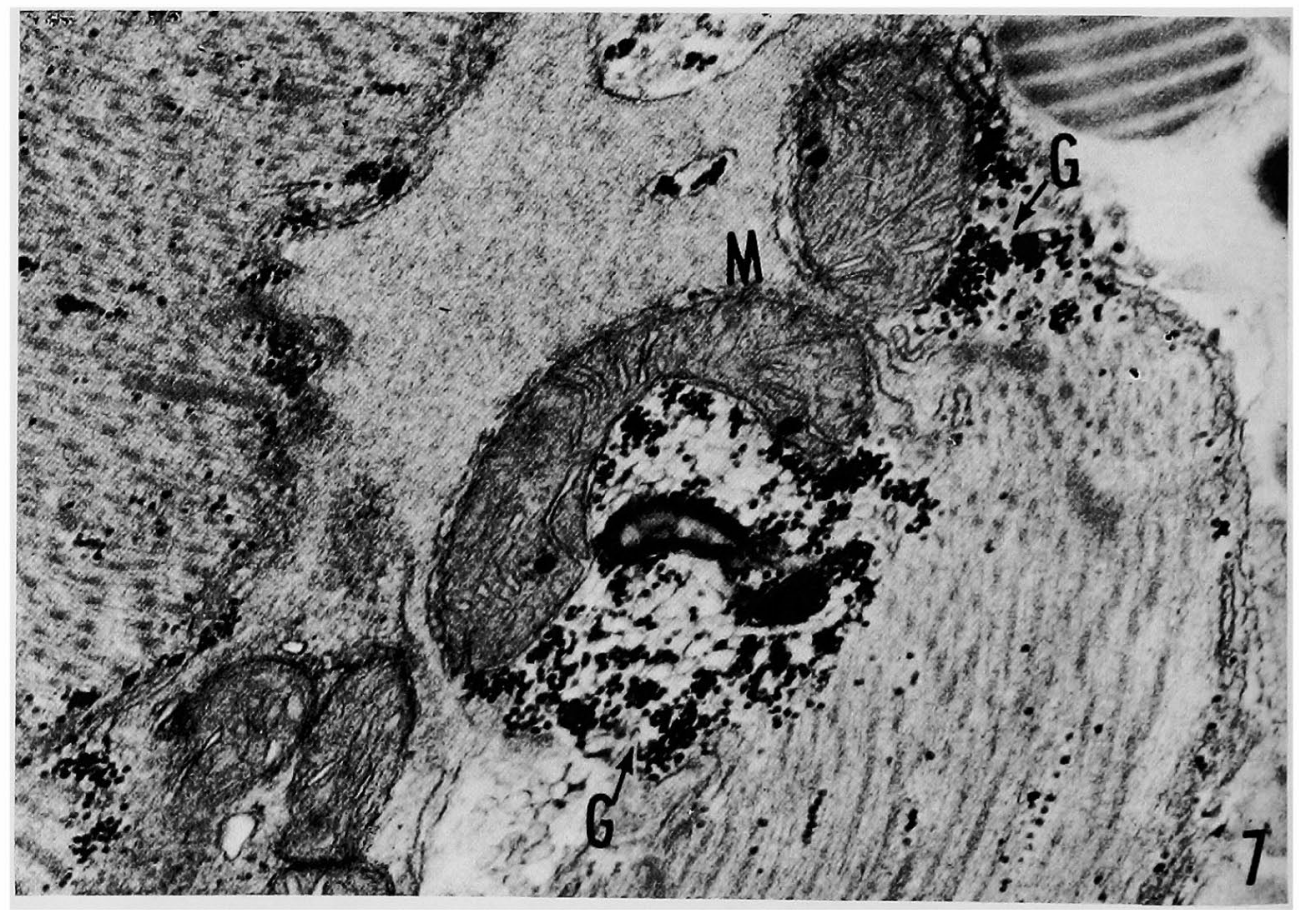

Fig. 7. Oblique sections of smooth muscle cells to show mitochondria (M) and glycogen granules (G). $\times 50,000$. 


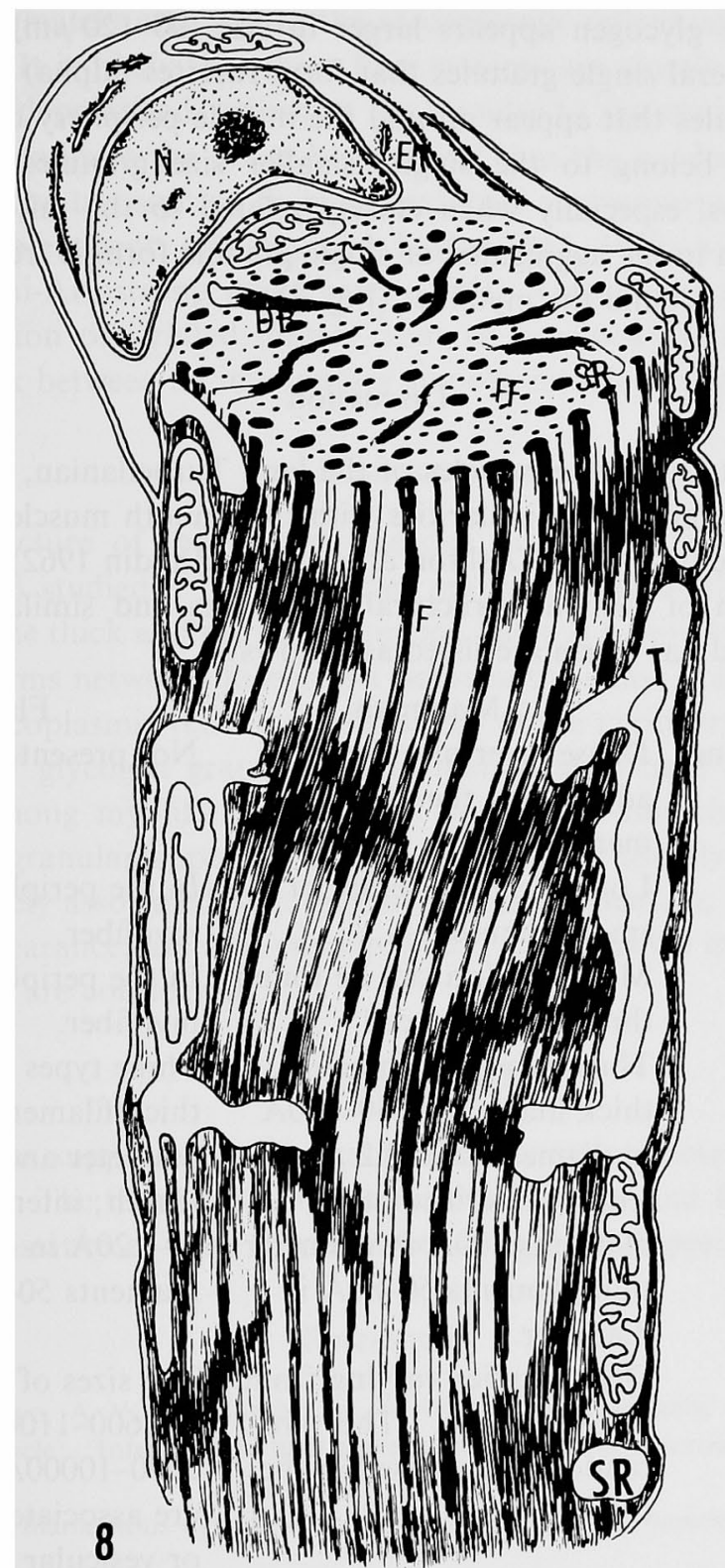

Fig. 8. Schematic drawing of a smooth muscle cell. DB, dense bodies; ER, endoplasmic reticulum; F, myofilament; M, mitochondrion; N, nucleus; SR, sarcoplasmic reticulum; T, tubular sarcoplasmic reticulum.

\section{Glycogen granules:}

The granules are considered to be glycogen based on their similarity to glycogen reported by other investigators (MacRae 1965). Glycogen is present in the periphery as well as between the myofilaments of the muscle fibers (Figs. 6, 7). Two types of glycogen can be distinguished from the electron micrographs. One type appears as single (beta) roughly spherical granules, about $30-40 \mu \mathrm{m}$ in diameter. 
The other type of glycogen appears larger in size $(90-120 \mu \mathrm{m})$, probably due to aggregation of several single granules that form rosettes (alpha) (Fig. 8). Most of the glycogen granules that appear around the muscle periphery or scattered among the myofilaments belong to the single, smaller size granules. Sometimes they cluster into groups, especially when closely related to the mitochondria (Fig. 7, arrow). Glycogen in the aggregated or single granule form is frequently associated with mitochondria around the myofiber (Fig. 2).

\section{Discussion}

The present study on the muscles of the land Turbellanian, Bipalium Kewense, indicates that there are some similarities with the smooth muscle of mammals, but there are also some differences (Ashton et al. 1975, Rhodin 1962).

A comparison of the fine structural differences and similarities of mammal smooth muscle and flat worm are listed as follows:

\section{Mammals}

Basement membrane Dense layer of material adjacent to the plasma membrane.

Nucleus

Mitochondria

Myofilaments

Dense bodies smooth muscle. throughout the cell. length; intermediate diameter.
Located in the center of the In the periphery of the Mitochondria can be found

Three types of filaments thick filaments, $150-250 \AA$ in diameter and $2.2 \mu \mathrm{m}$ in filaments $100 \AA$ in diameter; thin filaments $50-80 \AA$ in

Dense bodies varying from $700 \AA \times 4000 \AA$. They are randomly dispersed in the cytoplasm.

\section{Flat Worm}

Not present myofiber.

In the periphery of the myofiber.

Three types of filaments thick filaments $400-600 \AA$ in diameter and $1.5-3 \mu \mathrm{m}$ in length; intermediate filaments $80-120 \AA$ in diameter; thin filaments $50-60 \AA$ in diameter.

The sizes of the dense bodies are $600-1100 \AA$ wide and 5000-10000 $\AA$ long. They are associated with cisternae or vesicular of the sarcoplasmic reticulum.

Besides the two types of thick and thin myofilametns in flat worm smooth muscle, there is another type of intermediate filament. The dense bodies have been considered to be attachment sites of thin filaments analogous to the $\mathrm{Z}$ lines of straited muscles (Ashton et al. 1975, Schollmayer et al. 1973, Somlyo et al. 1971). The present study of flat worm, we find the thin filaments enter into the dense bodies. In agreement with the findings from mammalian smooth muscle by Ashton et al. (1975) and Cooke (1975), the intermediate filaments are also clearly associated with the dense bodies. This may suggest that there is some degree of order in the grouping of filaments in the contractile apparatus of smooth muscle cells. These 
bodies are also intimately attached to the sarcoplasmic reticulum system, as well as the sarcolemma. It is likely that these four systems are closely interrelated. The sarcolemma when depolarized, transmits the impulse to the sarcoplasmic reticulum which in turn transmits to the dense bodies, and thus the conducting electrical impulses would then be transmitted from the external environment into the fiber and to the contractable elements (Huxley and Taylor 1955, 1958) without benefit of a T system (Franzini-Armstrong and Porter 1964). One might therefore speculate that in the evolution of striated muscle, that we are looking, in the case of this organism, at a link between an involuntary smooth muscle and striated muscle.

\section{Abstract}

The fine structure of the smooth muscles in a land Turbellarian, Bipalium Kewense, has been studied. These muscles are composed of three types myofilaments. Besides the thick and the thin filaments, there is a third type of intermediate filament which forms networks to connect between the dense bodies. The nucleus, mitochondria, sarcoplasmic reticulum are mainly at the periphery of the muscle.

Two types of glycogen granules with different sizes have been encountered; both scattered among myofilaments as well as close to mitochondria. One type exhibits a round granular appearance while the other is usually of a rosette form. Dense bodies closely associated with the sarcoplasmic reticulum, which are vesicular or tubular in appearance, are also encountered. The possible evolutionary aspects of skeletal muscle are considered.

\section{Acknowledgements}

This work was supported by Veterans Administration Research funds, project number 8222-11. The technical assistance of Joe Meador and Bettye Stallings and the secretarial assistance of Mrs. Diane Butler are greatly appreciated.

\section{References}

Ashton, F. T., Somlyo, A. V. and Somlyo, A. P. 1975. The contractile apparatus of vascular smooth muscle: Intermediate high voltage stereo electron microscopy. J. Molec. Biol, 98: 17-29.

Cooke, P. 1975. A filamentous cytoskeleton in vertebrate smooth muscle fibers. J. Cell. Biol. 67: $78 \mathrm{a}$.

Franzini-Armstrong, C. and Porter, K. R. 1964. Sarcolemmal invaginations constituting the T system in fish muscle fibers. J. Cell Biol. 22: 675-696.

Hanson, J. and Lowy, J. 1960. Structure and function of the contractile apparatus in the muscles of invertebrate animals. In: G. H. Bourne (editor): Structure and Function of Muscle. Vol. 1, p. 265. Acad. Press Inc. London.

- and - 1961. The structure of the muscle fibers in the translucent part of the adductor of the oyster, Crassostrea angulata. Proc. Royal Society London, Series B, 154: 173.

Huxley, A. F. and Taylor, R. E. 1955. Function of Krause's membrane. Nature (London) 176: 1068.

- and - 1958. Local activation of striated muscle fibers. J. Physiol. (London) 144: 426-441.

Lusmgden, R. D. 1965. Macromolecular structure of glycogen in some cyclophyllidean and trypanorphyach cestodes. J. Parasitol. 51(4): 501-515. 
MacRae, E. K. 1963. Obseravations on the fine structure of pharyngeal muscle in the planarian dugesia tigrian. J. Cell Biol. 18: 651 .

- 1965. The fiber structure of muscle in a marine tubellarian. Zeitschrift für Zellforschung 68: 348-362.

North, R. J. 1963. The fine structure of the myofibers in the heart of snail Helix aspersa. J. Ultrastructure Res. 8: 206-218.

Philpott, D. E., Kahlbrock, M. and Szent-Gyorgyi, A. G. 1960. Filamentous organization of molluscan muscles. J. Ultrastructure Res. 3: 254-269.

Rhodin, J. A. G. 1962. Fine structure of vascular walls in mammals, with special reference to smooth muscle component. Physiological Review 42: 48-87.

Schollmayer, J. S., Goll, D. E., Robson and Stormer, M. H. 1973. Localization of $\alpha$-actinin and tropomyosin in different muscles. J. Cell Biol. 59: 306a.

Somlyo, A. P., Devine, C. E., Somlyo, A. V. and North, S. R. 1971. Sarcoplasmic reticulum and the temperature-dependent contraction of smooth muscle in calcium free solution. $J$. Cell Biol. 51 : 722-741. 